VoL. 43 (1991) [307-315]

\title{
AN IDENTITY INVOLVING NÖRLUND POLYNOMIALS
}

\author{
A.E. ÖzLüK AND C. SNYDER
}

We prove an identity involving Nörlund polynomials, the proof of which is elementary and involves the enumeration of lattice points. The identity is slightly stronger than an identity of Carlitz which he obtained by using Apostol's transformation formula for Lambert series.

\section{INTRODUCTION}

Using a transformation formula for Lambert series developed by Apostol [1], Carlitz [2], proved an identity from which he was able to derive a number of properties of Dedekind sums. Let us elaborate.

Let $m$ be a fixed odd integer with $m>1$ and let $j$ be an integer such that $0 \leqslant j \leqslant m+1$. Then the Dedekind sums of Apostol are defined as

$$
c_{j}(h, k)=\sum_{\mu(k)} \bar{B}_{m+1-j}\left(\frac{\mu}{k}\right) \bar{B}_{j}\left(\frac{h \mu}{k}\right)
$$

where $h, k$ are relatively prime integers, $\bar{B}_{n}(x)$ denotes the $n$th periodic Bernoulli polynomial, and the summation is over a complete set of residues modulo $k$.

More specifically, we define the $n$th Bernoulli polynomial by the series

$$
\sum_{n=0}^{\infty} B_{n}(x) \frac{z^{n}}{n !}=\frac{z e^{x z}}{e^{z}-1}
$$

Then the $n$th periodic Bernoulli polynomial is given by

$$
\bar{B}_{n}(x)=B_{n}(\{x\})
$$

for any real number $x$, where $\{x\}$ denotes the fractional part of $x$, that is $x-[x]$.

For $h, k, m$ as above and $\tau \in \mathrm{C}$, the set of complex numbers, with $\operatorname{Im} \tau>0$, define

$$
f(h, k ; \tau)=\sum_{j=0}^{m+1}\left(\begin{array}{c}
m+1 \\
j
\end{array}\right)(k \tau-h)^{m-j} c_{j}(h, k) .
$$

Received 3 May 1990

Copyright Clearance Centre, Inc. Serial-fee code: 0004-9729/91 \$A2.00+0.00. 
Carlitz then showed the identity

$$
f(-k, h ; \tau)=\tau^{m-1} f\left(h, k ;-\frac{1}{\tau}\right)+\frac{1}{\tau}(B+\tau B)^{m+1}
$$

where $(B+\tau B)^{m+1}$ is written symbolically for $\sum_{j=0}^{m+1}\left(\begin{array}{c}m+1 \\ j\end{array}\right) \tau^{j} B_{j} B_{m+1-j}$ and $B_{n}$ is the $n$th Bernoulli number defined by $B_{n}(0)$ where $B_{n}(x)$ is the $n$th Bernoulli polynomial.

Carlitz' identity is a consequence of Apostol's transformation formulas for Lambert series.

In this note, we prove a slightly stronger identity by reformulating it as an identity involving Nörlund polynomials. Our proof is elementary and ultimately depends on showing that two sets of lattice points are equal.

\section{DEFINITION AND BASIC PROPERTIES OF NÖRLUND POLYNOMIALS}

We now introduce the Nörlund polynomials and recall some of their properties; see [3] and [4] for some of the results.

DEFINITION: The $m$ th Nörlund polynomial in $n$ parameters $\omega_{1}, \ldots, \omega_{n}$, denoted $B_{m}^{(n)}\left(x ; \omega_{1}, \ldots, \omega_{n}\right)$, is defined by

$$
\frac{z^{n} e^{x z}}{\left(e^{\omega_{1} z}-1\right) \ldots\left(e^{\omega_{n} z}-1\right)}=\sum_{m=0}^{\infty} B_{m}^{(n)}\left(x ; \omega_{1}, \ldots, \omega_{n}\right) \frac{z^{m}}{m !}
$$

Here $\underline{\omega}=\left(\omega_{1}, \ldots, \omega_{n}\right) \in C^{n}$ and $x$ is a variable.

In particular

$$
\frac{z e^{x z}}{e^{\omega z}-1}=\sum_{m=0}^{\infty} B_{m}^{(1)}(x ; \omega) \frac{z^{m}}{m !}
$$

from which we see that

$$
B_{m}^{(1)}(x ; \omega)=\omega^{m-1} B_{m}\left(\frac{x}{\omega}\right)
$$

where $B_{m}(x)$ is the $m$ th Bernoulli polynomial given by

$$
\frac{z e^{x z}}{e^{z}-1}=\sum_{m=0}^{\infty} B_{m}(x) \frac{z^{m}}{m !}
$$

Proposition 1. For all nonnegative integers $m$ and $a \in C$,

$$
B_{m}^{(n)}\left(a x ; a \omega_{1}, \ldots, a \omega_{n}\right)=a^{m-n} B_{m}^{(n)}\left(x ; \omega_{1}, \ldots, \omega_{n}\right)
$$


Proof:

$$
\begin{aligned}
& \sum_{m=0}^{\infty} B_{m}^{(n)}(a x ; a \underline{\omega}) \frac{z^{m}}{m !}=\frac{z^{n} e^{a x z}}{\left(e^{a \omega_{1} z}-1\right) \ldots\left(e^{a \omega_{m} z}-1\right)} \\
& \quad=\frac{a^{-n}(a z)^{n} e^{x(a z)}}{\left(e^{\omega_{1}(a z)}-1\right) \ldots\left(e^{\omega_{n}(a z)}-1\right)}=\sum_{m=0}^{\infty} a^{m-n} B_{m}^{(n)}(x ; \underline{\omega}) \frac{z^{m}}{m !} .
\end{aligned}
$$

Proposition 2. For $m, n, \underline{\omega}$ as above, and $1 \leqslant j \leqslant n$,

$$
B_{m}^{(n)}(x ; \underline{\omega})=-B_{m}^{(n)}\left(x-\omega_{j} ; \omega_{1}, \ldots, \omega_{j-1},-\omega_{j}, \omega_{j+1}, \ldots, \omega_{n}\right) .
$$

Proof:

$$
\begin{aligned}
& \sum_{m=0}^{\infty} B_{m}^{(n)}(x ; \underline{\omega}) \frac{z^{m}}{m !}=\frac{z^{n} e^{x z}}{\left(e^{\omega_{1} z}-1\right) \ldots\left(e^{\omega_{n} z}-1\right)} \\
& \quad=\frac{z^{n} e^{x z} e^{-\omega_{j} z}}{\left(e^{\omega_{1} z}-1\right) \ldots\left(e^{\omega_{j} z}-1\right) e^{-\omega_{j} z} \ldots\left(e^{\omega_{n} z}-1\right)} \\
& \quad=\frac{z^{n} e^{\left(z-\omega_{j}\right) z}}{\left(e^{\omega_{1} z}-1\right) \ldots\left(1-e^{-\omega_{j} z}\right) \ldots\left(e^{\omega_{n} z}-1\right)} \\
& =\frac{-z^{n} e^{\left(x-\omega_{j}\right) z}}{\left(e^{\omega_{1} z}-1\right) \ldots\left(e^{-\omega_{j} z}-1\right) \ldots\left(e^{\omega_{n} z}-1\right)}=-\sum_{m=0}^{\infty} B_{m}^{(n)}\left(x-\omega_{j} ; \underline{\tilde{\omega}}\right) \frac{z^{m}}{m !}
\end{aligned}
$$

where $\underline{\widetilde{w}}=\left(\omega_{1}, \ldots, \omega_{j-1},-\omega_{j}, \omega_{j+1}, \ldots, \omega_{n}\right)$.

Proposition 3. Let $\left(n_{1}, \ldots, n_{k}\right)$ be a partition of $n$ and let $\underline{\omega}=\left(\omega_{1}, \ldots, \omega_{n}\right)$ $=\left(\underline{\omega}_{1}, \ldots, \underline{\omega}_{k}\right)$ where $\underline{\omega}_{1}=\left(\omega_{1}, \ldots, \omega_{n_{1}}\right), \ldots, \underline{\omega}_{k}=\left(\omega_{n-n_{k}+1}, \ldots, \omega_{n}\right)$. Then

$$
B_{m}^{(n)}\left(x_{1}+\ldots+x_{k} ; \underline{\omega}\right)=\left(B^{\left(n_{1}\right)}\left(x_{1} ; \underline{\omega}_{1}\right)+\ldots+B^{\left(n_{k}\right)}\left(x_{k} ; \underline{\omega}_{k}\right)\right)^{m}
$$

where the last expression is written symbolically for

$$
\sum_{\substack{j_{1}, \ldots, j_{k} \geqslant 0 \\ j_{1}+\ldots+j_{k}=m}} \frac{m !}{j_{1} ! \ldots j_{k} !} B_{j_{1}}^{\left(n_{1}\right)}\left(x_{1} ; \underline{\omega}_{1}\right) \ldots B_{j_{k}}^{\left(n_{k}\right)}\left(x_{k} ; \underline{\omega}_{k}\right) .
$$

Proof:

$$
\begin{aligned}
& \sum_{m=0}^{\infty} B_{m}^{(n)}\left(x_{1}+\ldots+x_{k} ; \underline{\omega}\right) \frac{z^{m}}{m !}=\frac{z^{n} e^{\left(x_{1}+\ldots+x_{k}\right) z}}{\left(e^{\omega_{1} z}-1\right) \ldots\left(e^{\omega_{n} z}-1\right)} \\
& \quad=\frac{z^{n_{1}} e^{x_{1} z}}{\left(e^{\omega_{1} z}-1\right) \ldots\left(e^{\omega_{n_{1} z} z}-1\right)} \cdots \frac{z^{n_{k}} e^{x_{k} z}}{\left(e^{\omega_{n-n_{k}+1^{z}}}-1\right) \ldots\left(e^{\omega_{n} z}-1\right)} \\
& \quad=\sum_{m=0}^{\infty}\left(B^{\left(n_{1}\right)}\left(x_{1} ; \underline{\omega}_{1}\right)+\ldots+B^{\left(n_{k}\right)}\left(x_{k} ; \underline{\omega}_{k}\right)\right)^{m} \frac{z^{m}}{m !}
\end{aligned}
$$




\section{An identity involving Nörlund Polynomials}

LEMMA. Let $h, k$ be relatively prime integers with $k>0$. Then

$$
f(h, k ; \tau)=\sum_{\mu=0}^{k-1} B_{m+1}^{(2)}\left(\tau \mu-\left[\frac{h \mu}{k}\right] ; k \tau-h, 1\right)
$$

and

$$
f(0,1 ; \tau)=B_{m+1}^{(2)}(0 ; \tau, 1)=\frac{1}{\tau}(B+\tau B)^{m+1} .
$$

Here $[x]$ is the largest integer less than or equal to $x$.

Proof:

$$
\begin{aligned}
& f(h, k ; \tau)=\sum_{j=0}^{m+1}\left(\begin{array}{c}
m+1 \\
j
\end{array}\right)(k \tau-h)^{m-j} c_{j}(h, k) \\
& =\sum_{j=0}^{m+1}\left(\begin{array}{c}
m+1 \\
j
\end{array}\right)(k \tau-h)^{m-j} \sum_{\mu=0}^{k-1} B_{m+1-j}\left(\frac{\mu}{k}\right) B_{j}\left(\left\{\frac{h \mu}{k}\right\}\right)
\end{aligned}
$$

where $\{x\}$ denotes the fractional part of $x$, that is, $x-[x]$. Since $B_{m}(x)=B_{m}^{(1)}(x ; 1)$, we see by Proposition 1 that

$$
\begin{aligned}
f(h, k ; \tau) & =\sum_{\mu=0}^{k-1} \sum_{j=0}^{m+1}\left(\begin{array}{c}
m+1 \\
j
\end{array}\right) B_{m+1-j}^{(1)}\left((k \tau-h) \frac{\mu}{k} ; k \tau-h\right) B_{j}^{(1)}\left(\left\{\frac{h \mu}{k}\right\} ; 1\right) \\
& =\sum_{\mu=0}^{k-1} \sum_{j=0}^{m+1}\left(\begin{array}{c}
m+1 \\
j
\end{array}\right) B_{m+1-j}^{(1)}\left(\tau \mu-\frac{h \mu}{k} ; k \tau-h\right) B_{j}^{(1)}\left(\left\{\frac{h \mu}{k}\right\} ; 1\right) .
\end{aligned}
$$

Then by Proposition 3 we have

$$
\begin{aligned}
f(h, k ; \tau) & =\sum_{\mu=0}^{k-1} B_{m+1}^{(2)}\left(\tau \mu-\frac{h \mu}{k}+\left\{\frac{h \mu}{k}\right\} ; k \tau-h, 1\right) \\
& =\sum_{\mu=0}^{k-1} B_{m+1}^{(2)}\left(\tau \mu-\left[\frac{h \mu}{k}\right] ; k \tau-h, 1\right) .
\end{aligned}
$$

In particular

$$
f(0,1 ; \tau)=B_{m+1}^{(2)}(0 ; \tau, 1) .
$$

But by Propositions 1 and 3 and the fact that $B_{m}=B_{m}(0)$, we see

$$
\begin{aligned}
B_{m+1}^{(2)}(0 ; \tau, 1) & =\sum_{j=0}^{m+1}\left(\begin{array}{c}
m+1 \\
j
\end{array}\right) B_{m+1-j}^{(1)}(0 ; \tau) B_{j}^{(1)}(0 ; 1) \\
& =\sum_{j=0}^{m+1}\left(\begin{array}{c}
m+1 \\
j
\end{array}\right) \tau^{m-j} B_{m+1-j} B_{j}=\frac{1}{\tau}(B+\tau B)^{m+1} .
\end{aligned}
$$


The lemma is now established.

By the lemma Carlitz' identity takes the form

$$
\begin{aligned}
& \sum_{\nu=0}^{h-1} B_{m+1}^{(2)}\left(\tau \nu-\left[\frac{-k \nu}{h}\right] ; h \tau+k, 1\right) \\
& \quad=\tau^{m-1} \sum_{\mu=0}^{k-1} B_{m+1}^{(2)}\left(-\frac{1}{\tau} \mu-\left[\frac{h \mu}{k}\right] ; k\left(-\frac{1}{\tau}\right)-h, 1\right)+B_{m+1}^{(2)}(0 ; \tau, 1)
\end{aligned}
$$

for all odd $m>1$. By using Proposition 1, this identity becomes

$$
\begin{aligned}
& \sum_{\nu=0}^{h-1} B_{m+1}^{(2)}\left(\tau \nu-\left[\frac{-k \nu}{h}\right] ; h \tau+k, 1\right) \\
& =\sum_{\mu=0}^{k-1} B_{m+1}^{(2)}\left(-\mu-\left[\frac{h \mu}{k}\right] \tau ;-(h \tau+k), \tau\right)+B_{m+1}^{(2)}(0 ; \tau, 1),
\end{aligned}
$$

for all odd $m>1$.

We establish a little more.

THEOREM. Let $h, k$ be relatively prime positive integers. Then for all integers $m \geqslant 0$,

$$
\begin{aligned}
& \sum_{\nu=0}^{h-1} B_{m}^{(2)}\left(\tau \nu-\left[\frac{-k \nu}{h}\right] ; h \tau+k, 1\right) \\
& =\sum_{\mu=0}^{k-1} B_{m}^{(2)}\left(-\mu-\left[\frac{h \mu}{k}\right] \tau ;-(h \tau+k), \tau\right)+B_{m}^{(2)}(0 ; \tau, 1) \\
& \quad+B_{m}^{(2)}(0 ; h \tau+k,-\tau)-B_{m}^{(2)}((h-1) \tau+k ; h \tau+k,-\tau)
\end{aligned}
$$

Corollary. Let $h, k$ and $m$ be as in the theorem. Then

$$
\begin{aligned}
& \sum_{\nu=0}^{h-1} B_{m}^{(2)}\left(\tau \nu-\left[\frac{-k \nu}{h}\right] ; h \tau+k, 1\right) \\
& =\sum_{\mu=0}^{k-1} B_{m}^{(2)}\left(-\mu-\left[\frac{h \mu}{k}\right] \tau ;-(h \tau+k), \tau\right)+B_{m}^{(2)}(0 ; \tau, 1) \\
& \quad+\left(1-(-1)^{m}\right) B_{m}^{(2)}(0 ; h \tau+k,-\tau) .
\end{aligned}
$$

REMARK. Notice that Carlitz' version is a special case when $m$ is even. ( $m$ above is $m+1$ in the Carlitz identity.) 
Proof of Corollary: By two applications of Proposition 2, we have

$$
\begin{aligned}
B_{m}^{(2)}((h-1) \tau+k ; h \tau+k,-\tau) & =-B_{m}^{(2)}(-\tau ;-(h \tau+k),-\tau) \\
& =B_{m}^{(2)}(0 ;-(h \tau+k), \tau) .
\end{aligned}
$$

Now apply Proposition 1 with $a=-1$ to obtain

$$
B_{m}^{(2)}(0 ;-(h \tau+k), \tau)=(-1)^{m-2} B_{m}^{(2)}(0 ; h \tau+k,-\tau)
$$

Proof of Theorem: We reformulate the theorem into a sequence of equivalent statements. We then prove the last of these statements by a lattice counting argument.

By the definition of the Nörlund polynomials, we need to show that

$$
\begin{array}{r}
\sum_{\nu=0}^{h-1} \frac{z^{2} e^{(\tau \nu-[-k \nu / k]) z}}{\left(e^{(h \tau+k) z}-1\right)\left(e^{z}-1\right)}-\sum_{\mu=0}^{k-1} \frac{z^{2} e^{(-\mu-[h \mu / k] \tau) z}}{\left(e^{-(h \tau+k) z}-1\right)\left(e^{\tau z}-1\right)} \\
-\frac{z^{2}}{\left(e^{\tau z}-1\right)\left(e^{z}-1\right)}+\frac{z^{2}\left(e^{((h-1) \tau+k) z}-1\right)}{\left(e^{(h \tau+k) z}-1\right)\left(e^{-\tau z}-1\right)}=0 .
\end{array}
$$

We factor out

$$
\frac{z^{2}}{\left(e^{(h r+k) z}-1\right)\left(e^{\tau z}-1\right)\left(e^{z}-1\right)}
$$

of the left-hand side of the equation and then divide both sides by this factor obtaining the equivalent statement

$$
\begin{aligned}
\left(e^{\tau z}-1\right) & \sum_{\nu=0}^{h-1} e^{(\tau \nu-[-k \nu / h]) z}+\left(e^{z}-1\right) e^{(h \tau+k) z} \sum_{\mu=0}^{k-1} e^{(-\mu-[h \mu / k] \tau) z} \\
& -\left(e^{(h \tau+k) z}-1\right)-e^{\tau z}\left(e^{z}-1\right)\left(e^{((h-1) \tau+k) z}-1\right)=0 .
\end{aligned}
$$

We now multiply out all the terms obtaining

$$
\begin{aligned}
& \sum_{\nu=0}^{h-1} e^{(\tau(\nu+1)-[-k \nu / h]) z}-\sum_{\nu=0}^{h-1} e^{(\tau \nu-[-k \nu / h]) z} \\
& \quad+\sum_{\mu=0}^{k-1} e^{((h-[h \mu / k]) \tau+k+1-\mu) z}-\sum_{\mu=0}^{k-1} e^{((h-[h \mu / k]) \tau+k-\mu) z} \\
& \quad-e^{(h \tau+k) z}+1-e^{(h r+k+1) z}+e^{(h r+k) z}+e^{(\tau+1) z}-e^{\tau z}=0 .
\end{aligned}
$$


We simplify the left-hand side and then divide both sides by $e^{\tau z}$ obtaining

$$
\begin{gathered}
\begin{array}{l}
\sum_{\nu=1}^{h-1} e^{(\tau \nu-[-k \nu / h]) z}-\sum_{\nu=1}^{h-1} e^{(\tau(\nu-1)-[-k \nu / h]) z}+\sum_{\mu=1}^{k-1} e^{((h-1-[h \mu / k]) \tau+k+1-\mu) z} \\
-\sum_{\mu=1}^{k-1} e^{((h-1-[h \mu / k]) \tau+k-\mu) z}-e^{((h-1) \tau+k) z}+e^{x}=0 .
\end{array} \\
\begin{aligned}
& \text { Using }-[-x]=\left\{\begin{array}{ll}
{[x]+1} & \text { if } x \notin Z \\
{[x]} & \text { if } x \in Z
\end{array}\right. \text { we get } \\
& \sum_{\nu=0}^{h-1} e^{(\tau \nu+[h \nu / k]+1) z}-\sum_{\nu=1}^{h-1} e^{(\tau(\nu-1)+[h \nu / k]+1) z}+\sum_{\mu=1}^{k-1} e^{((h-1-[(h / \mu) k]) \tau+k+1-\mu) z}
\end{aligned} \\
-\sum_{\mu=1}^{k-1} e^{(((h-1)-[h \mu / k]) \tau+k-\mu) z}-e^{((h-1) \tau+k) z}=0 .
\end{gathered}
$$

In the last two sums on the left-hand side, we change variable of summation $\mu \rightarrow$ $k-\mu$ and use $h-1-[h \mu / k] \rightarrow h-1-[h(k-\mu) / k]=h-1-h-[-(h \mu) / k]=$ $h-1-h+[h \mu / k]+1=[h \mu / k]$ for $1 \leqslant \mu \leqslant k-1$. Hence the above identity becomes

$$
\begin{gathered}
\sum_{\nu=0}^{h-1} e^{(\tau \nu+[k \nu / h]+1) z}-\sum_{\nu-1}^{h-1} e^{(\tau(\nu-1)+[k \nu / h]+1) z} \\
+\sum_{\mu=1}^{k-1} e^{([h \mu / k] \tau+\mu+1) z}-\sum_{\mu=1}^{k-1} e^{([h \mu / k] \tau+\mu) z}-e^{((h-1) \tau+k) z}=0 .
\end{gathered}
$$

We finally have an equivalent form of the theorem that we shall prove. Comparing terms shows that it suffices to prove

where

$$
\begin{aligned}
& S_{1} \dot{\cup} S_{2}=T_{1} \dot{\cup} T_{2} \dot{\cup} T_{3} \\
& S_{1}=\left\{\left(\nu,\left[\frac{k \nu}{h}\right]+1\right) \mid 0 \leqslant \nu \leqslant h-1\right\}, \\
& S_{2}=\left\{\left(\left[\frac{h \mu}{k}\right], \mu+1\right) \mid 1 \leqslant \mu \leqslant k-1\right\}, \\
& T_{1}=\left\{\left(\nu-1,\left[\frac{k \nu}{h}\right]+1\right) \mid 1 \leqslant \nu \leqslant h-1\right\}, \\
& T_{2}=\left\{\left(\left[\frac{h \mu}{k}\right], \mu\right) \mid 1 \leqslant \mu \leqslant k-1\right\}, \\
& T_{3}=\{(h-1, k)\} .
\end{aligned}
$$


Let

$$
\begin{aligned}
B & =\mathbf{Z} \times \mathbf{Z} \cap([0, h] \times[0, k]), \\
B_{0} & =\mathbf{Z} \times \mathbf{Z} \cap((0, h) \times(0, k)), \\
B_{1} & =\mathbf{Z} \times \mathbf{Z} \cap((0, h) \times[0, k]), \\
B_{2} & =\mathbf{Z} \times \mathbf{Z} \cap([0, h] \times(0, k)) .
\end{aligned}
$$

Also let $L$ denote the line in $\mathbf{R} \times \mathbf{R}$ with equation $y=(k / h) x$. Then geometrically we have

$$
\begin{aligned}
& S_{1}=\{P \in B \mid P \text { lies. just above } L\}, \\
& S_{2}=\left\{P \in B \mid\left(\exists Q \in B_{2}\right)(Q \text { lies just to the left of } L \text { and } P=Q+(0,1))\right\}, \\
& T_{1}=\left\{P \in B \mid\left(\exists Q \in B_{1}\right)(Q \text { lies just above } L \text { and } P=Q+(-1,0))\right\}, \\
& T_{2}=\left\{P \in B_{2} \mid P \text { lies just to the left of } L\right\} .
\end{aligned}
$$

Notice that from this description $S_{1}$ and $S_{2}$ are disjoint and $T_{1}, T_{2}, T_{3}$ are pairwise disjoint.

We now show that $S_{1} \cup S_{2}=T_{1} \cup T_{2} \cup T_{3}$. To this end, suppose $P \in S_{1} \cup S_{2}$.

CAse 1. Suppose $P \in S_{1}$. Then there are five possibilities which are depicted below. The points represent points in $B$ and the line segment represents a portion of $L$.
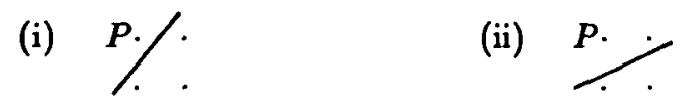
(iii) $P$. $(h, k)$
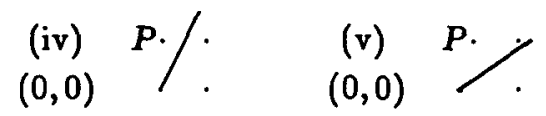

In (i) $P \in T_{2}$, in (ii) $P \in T_{1}$, in (iii) $P \in T_{3}$, in (iv) $P \in T_{2}$ and in (v) $P \in T_{1}$.

Case 2. Suppose $P \in S_{2}$. Then there are three possibilities given by
(i)

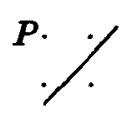
(ii) $\mathrm{P} . \mathrm{.}$
(iii) $P . /(h, k)$

In (i) $P \in T_{1}$, in (ii) $P \in T_{2}$ and in (iii) $P \in T_{3}$.

Thus $S_{1} \cup S_{2} \subseteq T_{1} \cup T_{2} \cup T_{3}$.

Now suppose $P \in T_{1} \cup T_{2} \cup T_{3}$.

CAse 1. Suppose $P \in T_{1}$. Then we have two possibilities:

(i) $P$.

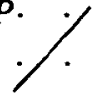

(ii)

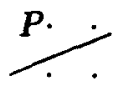

In (i) $P \in S_{2}$ and in (ii) $P \in S_{1}$. 
CASE 2. Suppose $P \in T_{2}$. Then we have two possibilities:
(i) $P . /$.
(ii) $\quad p \cdot \%$

In (i) $P \in S_{1}$ and in (ii) $P \in S_{2}$.

CASE 3. Suppose $P \in T_{3}$, that is, $P=(h-1, k)$. Then there are two possibilities:
(i) $P \cdot(h, k)$
(ii) $P . \rho^{(h, k)}$

In (i) $P \in S_{1}$ and in (ii) $P \in S_{2}$.

Therefore $T_{1} \cup T_{2} \cup T_{3} \subseteq S_{1} \cup S_{2}$.

The theorem is now established.

It would be of interest to see if there are similar identities involving Nörlund polynomials in more than two parameters. This might lead to the study of Lambert series in several variables as well as reciprocity laws for "multidimensional" Dedekind sums.

\section{REFERENCES}

[1] T. Apostol, 'Generalized Dedekind sums and transformation formulae of certain Lambert series', Duke Math. J. 17 (1950), 147-157.

[2] L. Carlitz, 'Some theorems on generalized Dedekind sums', Pacific J. Math. 3 (1953), 513-522.

[3] J. Kallies, 'Verallgemeinente Dedekindsche Summen und ein Gitterpunktproblem in n-dimensionalen Raum', J. Reine Angew. Math. 344 (1983), 22-37.

[4] N.E. Nörlund, Vorlesungen über Differenzrechnung (Berlin, 1924).

Department of Mathematics

Neville Hall

University of Maine

Orono, Maine 04469

United States of America 